\title{
Bidirectional Control of Anxiety-Related Behaviors in Mice: Role of Inputs Arising from the Ventral Hippocampus to the Lateral Septum and Medial Prefrontal Cortex
}

\author{
Gustavo Morrone Parfitt', Robin Nguyen', Jee Yoon Bang², Afif J Aqrabawi', Matthew M Tran ${ }^{2,3}$, \\ D Kanghoon Seo ${ }^{2,3}$, Blake A Richards ${ }^{2,3,4}$ and Jun Chul Kim*, I,2 \\ 'Department of Psychology, University of Toronto, Toronto, ON, Canada; ${ }^{2}$ Department of Cell \& Systems Biology, University of Toronto, Toronto, \\ ON, Canada; ${ }^{3}$ Department of Biological Sciences, University of Toronto Scarborough, Toronto, ON, Canada; ${ }^{4}$ Learning in Machines and Brains \\ Program, Canadian Institute for Advanced Research, Toronto, ON, Canada
}

\begin{abstract}
Anxiety is an adaptive response to potentially threatening situations. Exaggerated and uncontrolled anxiety responses become maladaptive and lead to anxiety disorders. Anxiety is shaped by a network of forebrain structures, including the hippocampus, septum, and prefrontal cortex. In particular, neural inputs arising from the ventral hippocampus (VHPC) to the lateral septum (LS) and medial prefrontal cortex (mPFC) are thought to serve as principal components of the anxiety circuit. However, the role of vHPC-to-LS and vHPC-to-mPFC signals in anxiety is unclear, as no study has directly compared their behavioral contribution at circuit level. We targeted LS-projecting VHPC cells and mPFC-projecting VHPC cells by injecting the retrogradely propagating canine adenovirus encoding Cre recombinase into the LS or mPFC, and injecting a Cre-responsive AAV (AAV8-hSyn-FLEX-hM3D or hM4D) into the VHPC. Consequences of manipulating these neurons were examined in well-established tests of anxiety. Chemogenetic manipulation of LS-projecting VHPC cells led to bidirectional changes in anxiety: activation of LS-projecting VHPC cells decreased anxiety whereas inhibition of these cells produced opposite anxietypromoting effects. The observed anxiety-reducing function of LS-projecting cells was in contrast with the function of mPFC-projecting cells, which promoted anxiety. In addition, double retrograde tracing demonstrated that LS- and mPFC-projecting cells represent two largely anatomically distinct cell groups. Altogether, our findings suggest that the vHPC houses discrete populations of cells that either promote or suppress anxiety through differences in their projection targets. Disruption of the intricate balance in the activity of these two neuron populations may drive inappropriate behavioral responses seen in anxiety disorders.
\end{abstract}

Neuropsychopharmacology (2017) 42, 17I5-1728; doi:I0.1038/npp.2017.56; published online 5 April 2017

\section{INTRODUCTION}

Anxiety is a temporary behavioral state induced by perceived threats (Sylvers et al, 2011). While it plays an adaptive role in protecting animals from danger, anxiety responses that are exaggerated or poorly controlled can be disruptive and are considered a core symptom of many psychiatric illnesses (Grupe and Nitschke, 2013). Animal models of anxiety have provided valuable insight into human anxiety disorders. In particular, rodent models of anxiety have proven informative. Anxiolytic drugs used in humans decrease anxietyrelated behaviors in rodents that are measured by innate avoidance of aversive environments (Calhoon and Tye, 2015). Thus, unraveling the neural circuitry of anxiety in rodents can help illuminate the mechanisms of anxiety disorders and identify potential treatment targets.

\footnotetext{
*Correspondence: Dr JC Kim, Department of Psychology, University of Toronto, 100 St George Street, Toronto, ON M5S3G3, Canada, Tel: + I 416978 4260, Fax: + I 416978 48। I,

E-mail: kim@psych.utoronto.ca

Received 22 September 2016; revised 27 February 2017; accepted 3 March 2017; accepted article preview online 15 March 2017
}

Gray and McNaughton proposed that the septohippocampal pathway is a crucial neurobiological circuit for controlling anxiety responses and a principal mediator of the behavioral effects of anxiolytic drugs (McNaughton and Gray, 2000; McNaughton, 2006). More specifically, its role was hypothesized to detect conflict and uncertainty in anxiety-provoking contexts and promote arousal, attention, and behavioral inhibition. Building upon this theoretical framework, it has long been postulated that hippocampal inputs to the septum carry contextual information regarding conflict, novelty, and uncertainty to control anxiety, although their behavioral contribution has not been directly investigated at the circuit level.

The septum and the hippocampus are extensively connected structures that form the septohippocampal pathway. The septum contains cholinergic, GABAergic and glutamatergic neurons that project to the hippocampus via the fimbria-fornix (Freund and Antal, 1988; Kiss et al, 1990; Wainer et al, 1985). The hippocampus, in turn, projects to the septum mainly via glutamatergic afferents (Niewiadomska et al, 2009; Risold and Swanson, 1997). Substantial evidence indicates that the septohippocampal 
pathway plays a major role in regulating anxiety. Disconnection of the lateral septum (LS) and the ventral hippocampus (vHPC) using an asymmetrical disconnection approach reduced anxiety-related behaviors in the elevated plus maze (EPM), suggesting that the vHPC and the LS work in tandem to modulate anxiety (Trent and Menard, 2010). When animals are subject to anxiety-provoking stimuli, the expression of activity-dependent protein $c$-fos is enhanced in both the septum and hippocampus (Campeau and Watson, 1997; Duncan et al, 1996; Mongeau et al, 2003; Pezzone et al, 1992; Senba et al, 1993; Silveira et al, 1993). However, the role of the septum in anxiety is complex. Septal lesions targeting both medial and LS lead to the well-known 'septal rage' phenotype in rodents, characterized by exaggerated or inappropriate defensive responses to nonthreatening stimuli (Albert and Walsh, 1982; Brady and Nauta, 1955). In contrast to these results, selective pharmacological inactivation of the LS reduces anxiety-related behaviors in the EPM and shock-probe burying test, and has suggested that this particular region of the septum may promote anxiety (Degroot et al, 2001; Menard and Treit, 1996; Pesold and Treit, 1996). Septal activation, however, has produced mixed results. Electrical stimulation of the LS reduced anxiety-related behaviors in the Vogel water-lick conflict test, while the activation of LS by corticotropinreleasing factor receptor 2 agonist infusion increased anxiety-related behaviors (Radulovic et al, 1999; Yadin et al, 1993). In resolving the complex role of the septum in anxiety, more selective techniques are required.

The second major component of the septohippocampal pathway that is involved in anxiety is the vHPC. Cytotoxic lesion or pharmacological inactivation of the vHPC, but not the dorsal hippocampus, reduces anxiety-related behaviors in rodents (Bannerman et al, 2003; Kjelstrup et al, 2002). Consistently, brain imaging studies in humans and rhesus monkeys have also found that elevated activity in the anterior hippocampus (equivalent to the vHPC in rodents) is associated with sustained anxiety (Hasler et al, 2007; Oler et al, 2010). Recent studies using in vivo LFP recordings and optogenetic inhibition revealed that vHPC input to the medial prefrontal cortex (mPFC) is necessary for the anxietypromoting role of the vHPC. Adhikari et al, 2011 found that vHPC theta oscillation is highly correlated with MPFC theta where theta power in both vHPC and mPFC increases in anxiety-inducing environments such as the EPM and open field (OF). These findings suggest that the vHPC promotes an anxious state by sending signals carrying the negative emotional valence of an environment to the mPFC (Adhikari et al, 2010, 2011). Consistently, a follow-up study from the same group found that optogenetic inhibition of vHPC input to the $\mathrm{mPFC}$ reduces anxiety and spatial representations of aversion within the mPFC (Padilla-Coreano et al, 2016).

Taken these various results together, it appears that the vHPC contributes to anxiety, likely by monitoring the novelty and aversiveness of the environment and sending contextual information to its downstream cortical and subcortical targets, including the septum and the prefrontal cortex. However, despite the established link between anxiety and forebrain circuits involving the vHPC, septum, and mPFC, the role of each pathway in controlling anxiety remains elusive, as no study has directly compared their behavioral contribution at the circuit level. Thus, our goal was to determine the role of VHPC inputs to the LS in regulating anxiety, and how they functionally and anatomically relate to the vHPC-mPFC pathway.

To this end, the present study used chemogenetic techniques to selectively manipulate the activity of individual vHPC cell populations projecting to the LS or mPFC during well-established tests of anxiety. We found that hM3Dmediated activation of the LS-projecting vHPC cells decreased anxiety-related behaviors, whereas hM4Dmediated inhibition of these cells produced the opposite anxiety-promoting effect. hM3D-mediated activation and double retrograde tracing revealed that these LS-projecting vHPC cells represent an anatomically and functionally distinct neuron population, largely separate from mPFCprojecting vHPC cells, activation of which increased anxiety.

\section{MATERIALS AND METHODS}

\section{Animals}

Adult C57BL/6 mice (3-4 month old) purchased from Charles River laboratories were housed in groups of four per cage without environmental enrichment in a temperaturecontrolled room (12:12 light/dark cycle), and food and water were provided ad libitum. One week after the arrival in the facility, animals were divided into control and treated groups and underwent stereotaxic surgery for viral infusion. A recovery period of 4 weeks was given before the beginning of the behavioral test to allow viral expression of hM3D and hM4D. The hM3D and hM4D expression for all the subjects were confirmed by immunohistochemistry signals for mCherry reporter as reference. Mice exhibiting low-level viral expression ( $<100$ labeled cells) or viral expression outside the vHPC were excluded from further analysis. The same group of mice were tested in either three (OF, EPM, successive alley (SA)) or four (OF, EPM, SA, NSF) anxiety tests. The order of first three tests (OF, EPM, SA) was counterbalanced. To further minimize carryover effects, the novelty suppressed feeding test (NSF) was performed last as it involved a food deprivation. All procedures were performed in accordance with the Canadian Council of Animal Care guidelines at the University of Toronto with an approved animal protocol.

\section{Stereotaxic Surgery and Viral Vectors}

Mice were anaesthetized with isoflurane (4\% for induction and $2 \%$ for maintaining) together with an injection of ketoprofen $5 \mathrm{mg} / \mathrm{kg}$ for pain management. For the viral infusion, a cannula connected to polyethylene tubing was inserted in the target brain region and left for $2 \mathrm{~min}$ to stabilize. After stabilization, a $0.1 \mu \mathrm{l}$ per minute infusion was performed followed by a $15 \mathrm{~min}$ resting period to permit the vector diffusion. Approximately $0.3-0.5 \mu \mathrm{l}$ of viral vectors $\left(\sim 10^{12} \mathrm{GC} / \mathrm{ml}\right)$ were bilaterally injected into the dorsal LS (+0.5 AP, $0.35 \mathrm{ML}$ and 2.7 DV; CAV2-Cre) or prelimbic mPFC (+1.9 AP, 0.3 ML and 1.9 DV; CAV2-Cre) and in the vHPC (-2.9 AP, 2.0 ML and 4.6 DV; AAVs) using a microsyringe pump. After the infusion, the cannula was slowly removed and the scalp sutured. AAV8-CaMKII $\alpha$ hM4D-mCherry and AAV8-CaMKII $\alpha$-GFP were used to exclude local inhibitory interneurons and target selectively 
excitatory cells in the vHPC. CAV2-Cre, AAV8-hSyn-FLEXGFP, AAV8-hSyn-FLEX-hM4D-mCherry, and AAV8-hSynFLEX-hM3D-mcherry were used to target LS-projecting vHPC cells and mPFC-projecting vHPC cells. A combined use of retrograde CAV-Cre and hSyn promoter-driven AAV was sufficient to exclude transgene expression in local interneurons. All AAVs were obtained from the University of North Carolina at Chapel Hill Vector Core. CAV2-CRE vector was obtained from the Plateforme de Vectorologie de Montpellier.

\section{Stereotaxic Surgery for the Anterograde Tracing Experiments}

Adult C57BL/6 inbred mice (3-4 month old) underwent stereotaxic surgery to infuse AAV-hsyn-ChR2-EYFP (channel rhodopsin fused to EYFP and driven by the human synapsin promoter) into the vHPC. Before the stereotaxic procedure, mice were anaesthetized with isoflurane (4\% for induction and $2 \%$ for maintaining) together with an injection of ketoprofen $5 \mathrm{mg} / \mathrm{kg}$ for pain management. For the viral infusion, a cannula connected to polyethylene tubing was inserted in the target brain region and left for $2 \mathrm{~min}$ to stabilize. After stabilization, a $0.1 \mu \mathrm{l}$ per minute infusion was performed followed by a $15 \mathrm{~min}$ resting period to permit the vector diffusion. The viral vector $\left(\sim 10^{12} \mathrm{GC} / \mathrm{ml}\right)$ was bilaterally injected into the vHPC (-2.9 AP, 2.0 ML and 4.6 DV) using a microsyringe pump. After the infusion, the cannula was slowly removed and the scalp sutured. A period of 4 weeks was given before the perfusion and histology procedure.

\section{Drugs}

Clozapine-N-oxide (CNO) was obtained from the $\mathrm{NIH}$ as a part of the Rapid Access to Investigative Drug Program funded by the National Institute of Neurological Disorders and Stroke (NINDS) and dissolved in DMSO (a final concentration of $0.3 \%$ DMSO). The animals were injected intraperitoneally with CNO 20 min before all behavioral tests at $1 \mathrm{mg} / \mathrm{kg}$ dose for hM4D experiments or at $3 \mathrm{mg} / \mathrm{kg}$ dose for hM3D experiments. CNO doses were determined based on the dose-response curves (CNO dose vs anxiety-related behaviors measured in the open field test).

\section{Behavioral Tasks}

Open field test. Animals were injected intraperitoneally with CNO (1 or $3 \mathrm{mg} / \mathrm{kg}$ ) and then placed in the testing room for a $20 \mathrm{~min}$ habituation. The OF apparatus consisted of a $50 \times 50 \times 50 \mathrm{~cm}^{3}$ Plexiglas chamber with the center area fined as a $30 \times 30 \mathrm{~cm}^{2}$ square. At the start of the test, animals were placed in a corner. The test lasted $20 \mathrm{~min}$ and was conducted under red light illumination. The Anymaze software (Stoelting, Wood Dale, IL) was used to track behavioral parameters.

Elevated plus maze test. The animals were injected intraperitoneally with CNO ( 1 or $3 \mathrm{mg} / \mathrm{kg}$ ), and then placed in the testing room for a $20 \mathrm{~min}$ habituation. The EPM apparatus consists of two black plastic open arms $(30 \mathrm{~cm} \times 5 \mathrm{~cm})$ perpendicularly conjoined at a center $\left(5 \times 5 \mathrm{~cm}^{2}\right)$ with two plastic enclosed arms $\left(30 \times 5 \times 30 \mathrm{~cm}^{3}\right)$. The maze was elevated one meter from the ground. Animals were placed in the center of the apparatus facing the open arms and allowed freely explore for $5 \mathrm{~min}$ under red light illumination. The Anymaze software (Stoelting, Wood Dale, IL) was used to track behavioral parameters.

Novelty suppressed feeding test. Before testing, mice were deprived of food for a period of $24 \mathrm{~h}$. During the test, animals were injected with vehicle or $\mathrm{CNO}$ and placed in the testing room for a $20 \mathrm{~min}$ habituation. After the habituation, animals were placed in an open-field arena with a $\sim 5 \mathrm{~g}$ regular food pellet on an aluminum foil situated in the arena center, and the latency to feed was manually recorded. The test was repeated after 1 week, and the treatments were counterbalanced between the groups. Immediately after the test, the animals were placed back in their home cages and allowed to eat for $5 \mathrm{~min}$, and the total food consumption was recorded. The NSF tests were conducted with a light (100 lux) placed $1.5 \mathrm{~m}$ above the arena for the vHPC-LS hM3D activation experiments to avoid the floor effect, and in a low light environment for the vHPC-mPFC hM3D activation experiments to avoid the ceiling effect. This design was driven by our hypothesis that activating the LS-projecting vHPC cells would decrease the latency to eat, whereas activating the mPFC-projecting vHPC cells would increase the latency to eat.

Successive alleys. The successive alleys (SUA) apparatus consists of four successive linearly connected alleys (alley 1-4) (Deacon, 2013; McHugh et al, 2004). The first alley (alley 1) is enclosed and painted in black, and is followed by three light colored open alleys (alley 2-4). The width of the four alleys progressively decreases to increase their anxiogenic character. The animals were placed in the enclosed alley and allowed to explore the apparatus for $10 \mathrm{~min}$. The Anymaze tracking system was used to track and acquire behavioral measurements. The same within-subjects design as that used in the NTS test was adopted in the SUA test.

\section{Immunohistochemistry}

Animals were transcardially perfused with ice-cold phosphate-buffered saline (PBS) ( $\mathrm{pH}$ 7.4) followed by paraformaldehyde $4 \%$. After perfusion, the brains were extracted and post-fixed in $4 \%$ paraformaldehyde for $24 \mathrm{~h}$ and then transferred to a $30 \%$ sucrose solution for $24 \mathrm{~h}$. Brains were sectioned coronally ( $40 \mu \mathrm{m}$ sections) using a cryostat (Leica) and collected in PBS. The sections were permeabilized using $0.5 \%$ triton/PBS (PBST) for $5 \mathrm{~min}$ and then blocked with $5 \%$ normal donkey serum in PBST for $1 \mathrm{~h}$. After blocking, the sections were washed three times with PBST and incubated overnight in rabbit polyclonal anti-mCherry antibody (1:1000; ab167453; Abcam, Cambridge, MA) or rabbit polyclonal anti-CRE antibody $(1: 1000$; ab167453; Abcam, Cambridge, MA). After incubation with the primary antibody, the sections were washed three times with PBST and incubated with Alexa 594-conjugated donkey anti-rabbit (1:1000; 715515152; Jackson ImmunoResearch, West Grove, PA) or Alexa 488-conjugated donkey anti-rabbit (1:1000; 711545152; Jackson ImmunoResearch, West Grove, PA) 
secondary antibodies for $1 \mathrm{~h} 30 \mathrm{~min}$. The sections were counterstained with $4^{\prime}$,6-diamidino-2- phenylindole (DAPI) for $10 \mathrm{~min}$ and coverslipped with Aquamount (Polysciences Inc, Warrington, PA). The wide-field fluorescent images were captured with the fluorescence microscope (Olympus FSX100) using an U-MWIG3 323 filter cube (Ex530-550, Em575IF, DM570) for Alexa 594. The confocal images were acquired through a Quorum spinning disk confocal micro-

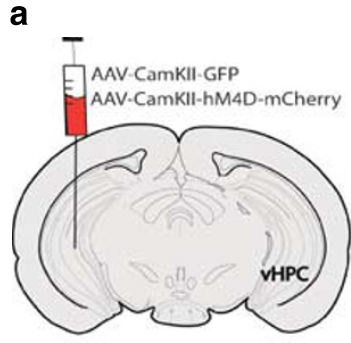

b

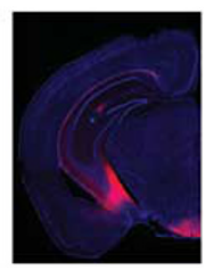

Bregma $^{-2.1} \mathrm{~A}$
mCherry/DAPI

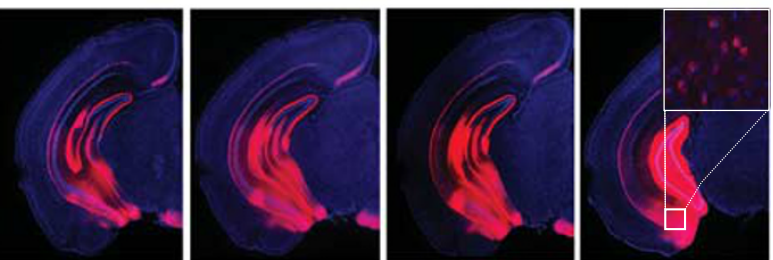

$-3.4$
C

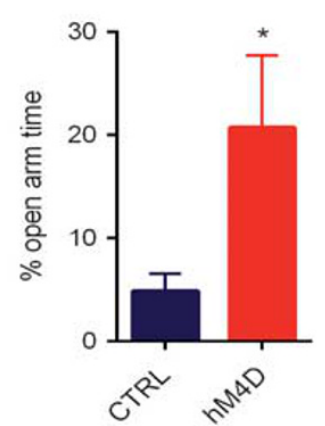

g

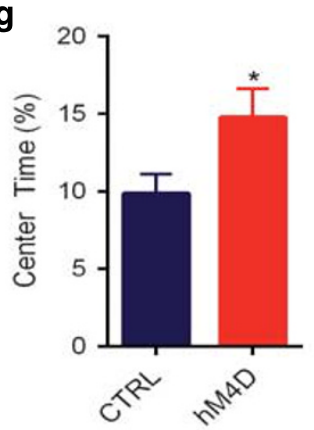

d

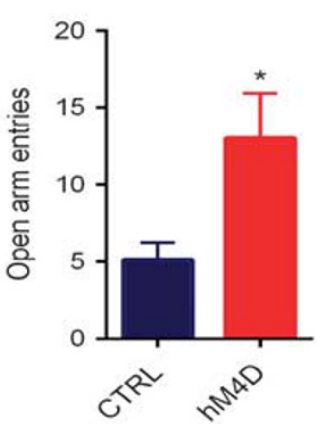

h

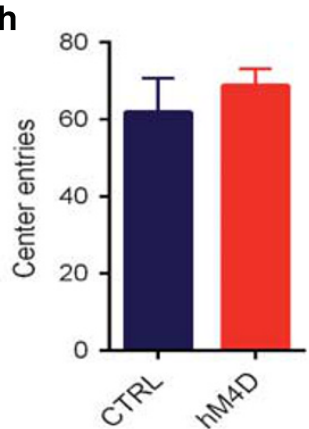

k
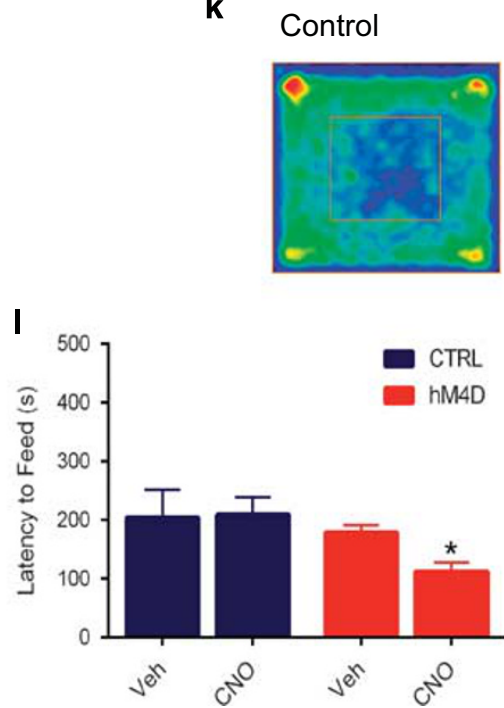

e

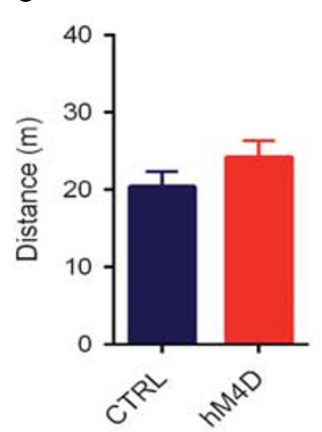

i

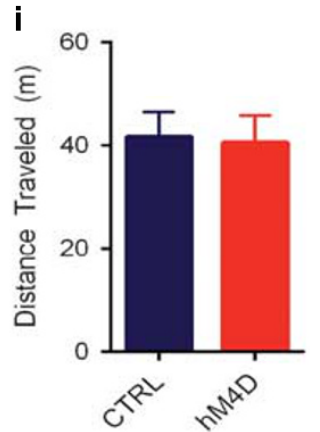

j

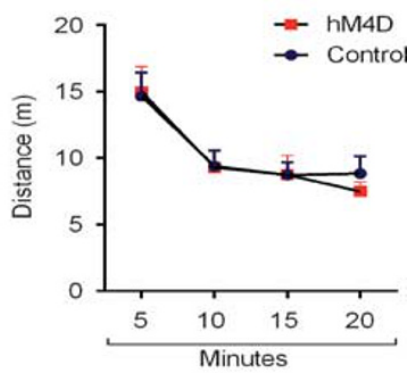

hM4D
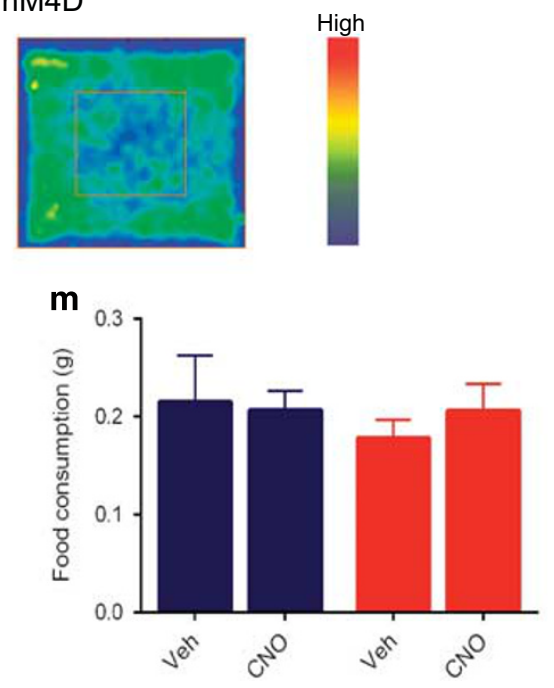
scope (Zeiss) using a $\times 20$ objective lens and were subsequently analyzed with the Image J software.

\section{Retrograde Tracing}

Dual retrograde tracing was performed to visualize cells in the vHPC that innervate the LS and mPFC. Green fluorescent Retrobeads (\# G180, Lumafluor Inc, Naples, FL) and Red fluorescent Retrobeads (\# R180, Lumafluor Inc, Naples, FL) were infused ipsilaterally at a volume of $0.5 \mu \mathrm{l}$ into the LS (+0.5 AP, $0.35 \mathrm{ML}$, and 2.7 DV) and mPFC $(+1.9$ AP, $0.3 \mathrm{ML}$, and 1.6 DV), respectively. Two weeks after surgery, mice were transcardially perfused, and brains were sectioned, as described in the immunohistochemistry procedure. Every fourth section containing the vHPC from bregma -2.90 to -3.85 , for a total of six sections per brain, were mounted and imaged on a Quorum spinning disk confocal microscope (Zeiss) using a $\times 20$ objective lens. The images were analyzed with the Volocity software (PerkinElmer) to count red and/or green retrobead-positive cells in a $1500 \mu \mathrm{m}$ length of the pyramidal layer in the ventral hippocampus. The percentage overlap for each red or green-labeled population was calculated as (total number of double-labeled cells/total number of single-labeled cells) $\times 100$.

\section{Hippocampal Slice Electrophysiology}

Following viral infusion of hM4D-mCherry or hM3DmCherry and 3-4 weeks wait time for expression, mice were anaesthetized with avertin and underwent cardiac perfusion with chilled, oxygenated cutting solution, containing the following (in mM): 205 sucrose, $2.5 \mathrm{KCl}, 1.25 \mathrm{NaH}_{2} \mathrm{PO}_{4}, 25$ $\mathrm{NaHCO}_{3}, 25$ glucose, 0.4 ascorbic acid, $1 \mathrm{CaCl}_{2}, 2 \mathrm{MgCl}_{2}$, and 3 sodium pyruvate, $\mathrm{pH} 7.4$, osmolality $290-295 \mathrm{mOsm}$. Immediately after perfusion, the mice were decapitated and the brains were rapidly removed and placed for $30 \mathrm{~s}$ in oxygenated cutting solution. Afterwards, the brains were cut at the cerebellum and glued on their posterior edge to a cutting stage. Coronal slices $(350 \mu \mathrm{m})$ of the ventral hippocampus were prepared and left to incubate at $36{ }^{\circ} \mathrm{C}$ in an oxygenated 50:50 mixture composed of cutting saline and artificial CSF (aCSF) for $30 \mathrm{~min}$. After this incubation the slices were and then placed in aCSF alone for $30 \mathrm{~min}$ at room temperature. During experimentation slices were perfused at a rate of $2 \mathrm{ml} / \mathrm{min}$ in aCSF maintained at $32^{\circ} \mathrm{C}$ via an in-line heater. The aCSF solution consisted of the following (in mM): $123 \mathrm{NaCl}, 2.5 \mathrm{KCl}, 1.25 \mathrm{NaH}_{2} \mathrm{PO}_{4}, 25$ $\mathrm{NaHCO}_{3}, 25$ glucose, $2 \mathrm{CaCl}_{2}$, and $1 \mathrm{MgCl}_{2}$ in double- distilled water and saturated with $95 \% \mathrm{O}_{2} / 5 \% \mathrm{CO}_{2}, \mathrm{pH}$ 7.4, osmolarity 290-295 mOsm. Whole-cell patch-clamp recordings were obtained from infected neurons (identified by mCherry fluorescence) and their uninfected neighbors. Micropipettes with a tip resistance of 3-6 M $\Omega$ were filled with an intracellular fluid containing the following (in $\mathrm{mM}$ ): 130 potassium gluconate, $10 \mathrm{KCl}, 10$ HEPES, 0.2 EGTA, 4 ATP, 0.3 GTP, and 10 phosphocreatine, pH 7.4, osmolality 290-295 mOsm. The resting membrane potential was recorded (in current-clamp mode) in normal aCSF for a minimum of $5 \mathrm{~min}$ before the addition of $10 \mu \mathrm{M}$ CNO for $5 \mathrm{~min}$. Neuronal excitability was determined in currentclamp mode by injecting a positive current $(100 \mathrm{pA})$ for $2 \mathrm{~s}$ (Nguyen et al, 2014).

\section{Statistical Analysis}

The statistical analysis was performed using the Graphpad Prism 6 (GraphPad Software, La Jolla, CA). For the EPM and OF tests, the statistical analysis for the comparisons was performed using the two-tailed unpaired $t$-test. For the NSF and SUA tests employing a within-subjects design, the data were subjected to a repeated-measures (RM) two-way ANOVA followed by Bonferroni post-test. All the data are presented as mean \pm standard error, and the difference was considered significant when $p<0.05$.

\section{RESULTS}

\section{Chemogenetic Inhibition of vHPC Decreases Anxiety}

To investigate the role of vHPC in controlling anxiety, we expressed inhibitory hM4D receptor-mCherry fusion protein in vHPC using an adeno-associated viral (AAV) vector under the control of the CaMKII $\alpha$ promoter (AAV8CaMKII $\alpha$-hM4D-mCherry) to target CaMKII $\alpha$-positive excitatory cells (Figure 1a and b). Age-matched control animals were infused with the same viral vector carrying GFP reporter gene (AAV8-CaMKII $\alpha$-GFP). In-line with previous studies (Armbruster et al, 2007; Nguyen et al, 2014), whole-cell patch-clamp recordings on hippocampal slices confirmed that synthetic ligand $\mathrm{CNO}$ activated hM4D receptor, hyperpolarizing membrane potential and suppressing current-induced spike activity in hippocampal pyramidal cells (Supplementary Figure S1). Four weeks after viral infection, the effect of acute CNO injection $(3 \mathrm{mg} / \mathrm{kg}$ i.p) on anxiety-related behaviors was analyzed using the EPM and OF test. In the EPM test, CNO-treated hM4D mice displayed an increase in \% open arm time (Unpaired $t$ test, $t_{(14)}=2.2$,

\footnotetext{
Figure I hM4D-mediated inhibition of the vHPC decreases anxiety. (a) AAV8-CaMKIl $\alpha$-hM4D-mCherry or AAV8-CaMKIll $\alpha$-GFP (control; CTRL) was injected into the VHPC bilaterally. (b) Representative images from AAV8-CaMKIl $\alpha$-hM4D-mCherry mice showed hM4D-mCherry expression in the vHPC along the anterior-posterior axis. (c) hM4D mice displayed an increased \% open arm time in the EPM test after CNO injection (* $p=0.04$ ). (d) hM4D mice displayed increased open arm entries in the EPM test after CNO injection (*p=0.0I). (e) CTRL and hM4D mice showed similar levels of locomotor activity measured as a total distance traveled in the EPM after CNO injection. (f) Heat-map plots show the averaged cumulative time spent in different parts of the EPM. red $=$ more time, blue = less time. (g) hM4D-mice displayed an increase in \% time spent in the center during the OF test (*p $=0.02$ ). (h and i) CTRL and hM4D mice showed similar levels of center entries (h) and locomotor activity (i) in the OF test after CNO injection. (j) Examination of locomotor activity in 5 min intervals revealed no significant differences between hM4D and CTRL mice. ( $k$ ) Heat-map plots show the averaged cumulative time spent in different parts of the OF. red = more time, blue = less time. $n=7$ for CTRL and 8 for hM4D mice. (I) CNO decreased the latency to feed in hM4D mice but not in CTRL mice in the NSF test (RM two-way ANOVA shows a significant effect of the treatment $F_{(1,9)}=5.344$ * $p=0.04$, followed by Bonferroni post-test $p=0.0 \mathrm{I}) .(\mathrm{m})$ No alteration in the food consumption was observed among the groups after the NSF test. $n=6$ for CTRL and 5 for hM4D mice.
} 
$p=0.04$, Figure $1 \mathrm{c}$ and $\mathrm{f}$ for position plots) and open arm entries compared to CNO-treated control group (Unpaired $t$ test, $t_{(14)}=2.5, p=0.02$, Figure $1 \mathrm{~d}$ ), with no change in locomotion (Figure 1e). These results suggested that hM4D-mediated inhibition of vHPC excitatory cells reduced anxiety in the EPM. Similarly, in the OF test, CNO-treated hM4D mice showed an increase in time spent in the center of the open field (Unpaired $t$ test, $t_{(14)}=2.54, p=0.02$, Figure $1 \mathrm{~g}$ and $\mathrm{k}$ for position plots) compared to CNO-treated control mice, and no change in the center entries (Figure 1h). The

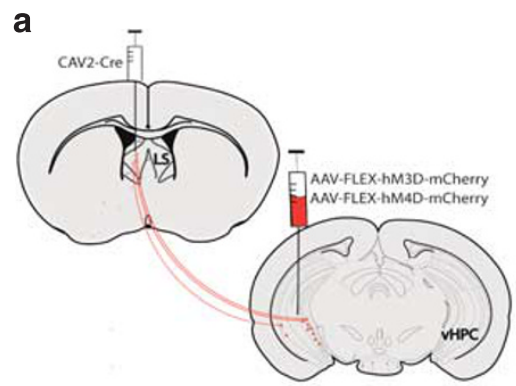

b
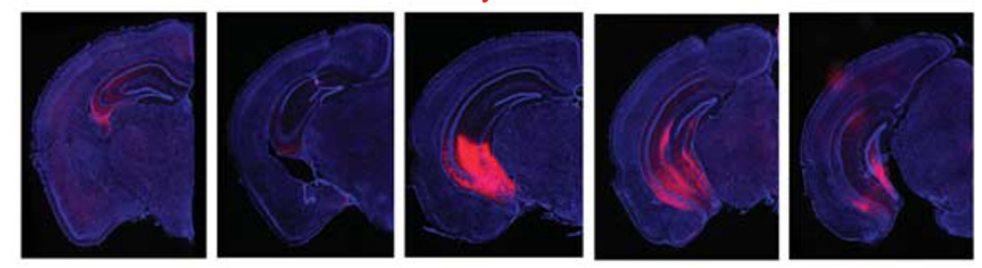

Bregma $^{-2.1}$ d

\section{C}
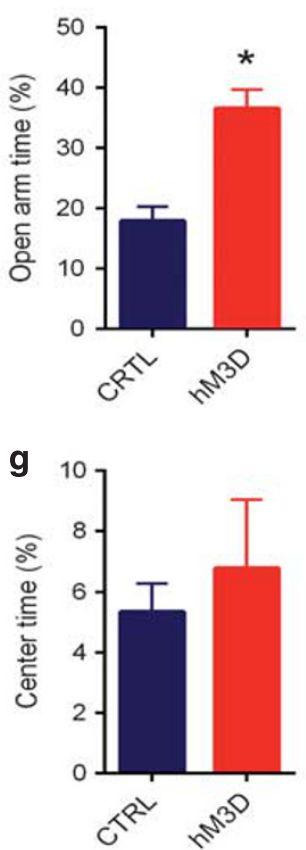

h
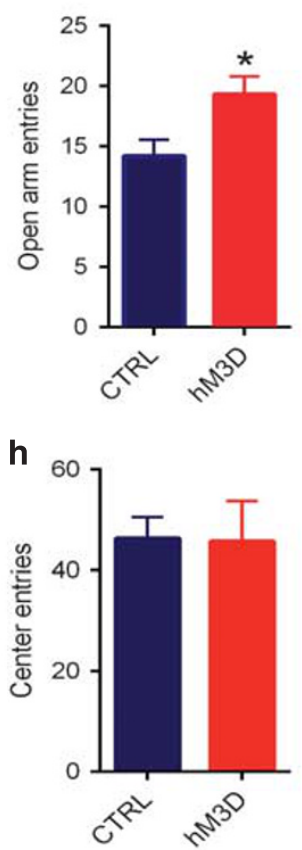

k Control
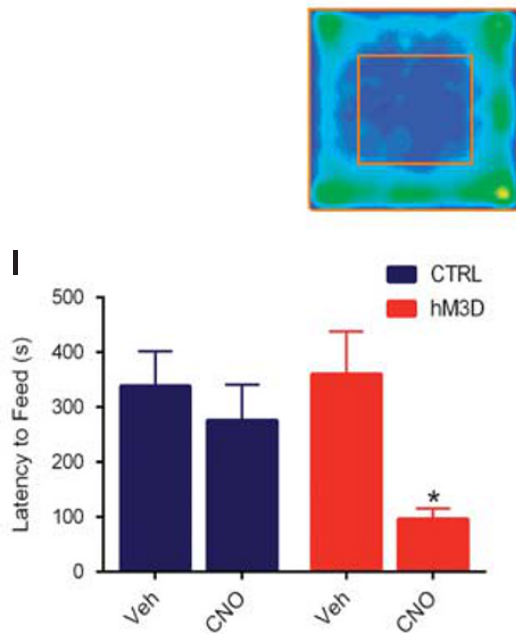

e

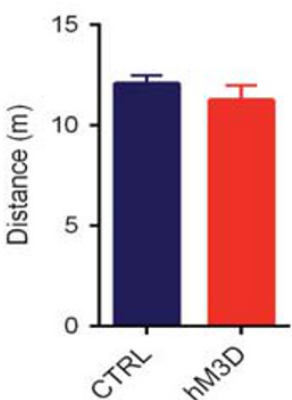

i

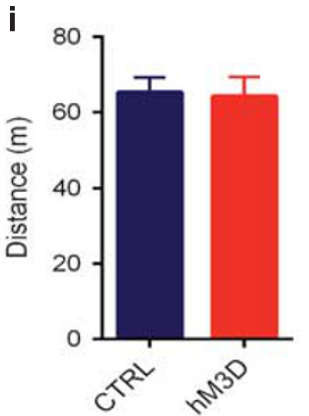

j

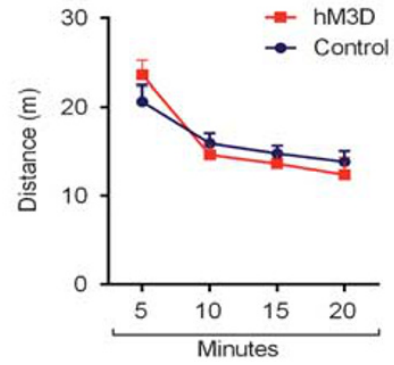

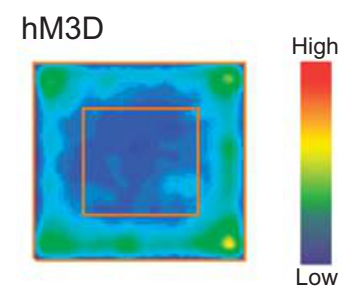

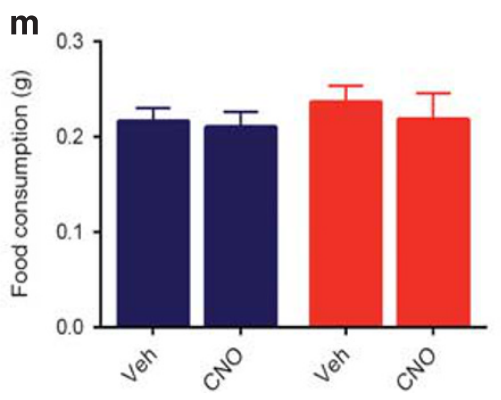


total distance traveled in the open field was not altered by the vHPC inhibition, excluding the possibility that the observed effect on anxiety was due to change in overall locomotion (Figure $1 \mathrm{i}$ and $\mathrm{j}$ ). In addition, the NSF was performed as a complementary test to measure anxiety-related behaviors. In the NSF test, CNO-treated hM4D mice showed reduced latency to feed in a novel environment (RM two-way ANOVA, $\mathrm{F}_{(1,9)}=5.344 p=0.04$, Figure 11$)$. The reduced latency to feed was not due to changes in hunger, since hM4D and control mice showed no difference in home cage food consumption (Figure $1 \mathrm{~m}$ ). Thus, the net effect of inhibiting glutamatergic outputs from the vHPC is a decrease in anxiety.

\section{hM3D-Mediated Activation of LS-Projecting vHPC Cells Decreases Anxiety}

The vHPC may shape anxiety via influences on other brain structures. The vHPC innervates the LS, a structure critically involved in anxiety, via the septohippocampal pathway. Previous studies, as well as our anterograde tracing experiments (Supplementary Figure S2), have shown that the LS is one of the most densely innervated targets of the vHPC (Risold and Swanson, 1997). To address the behavioral contribution of the vHPC cells projecting to the LS in anxiety, we bilaterally infused the retrogradely propagating canine adenovirus encoding Cre recombinase (CAV2-Cre) into the LS, followed by a bilateral vHPC infusion of AAV vector carrying a Cre recombinasedependent excitatory hM3D-mCherry construct (AAV8hSyn-FLEX-hM3D-mCherry) (Figure 2a) (Armbruster et al, 2007; Kremer et al, 2000). This allowed us to express excitatory hM3D receptors specifically in vHPC cells that project to the LS, which we here designate as LS-projecting vHPC cells. Local spread of CAV2-Cre virus within the LS was evaluated by immunostaining for Cre, which confirmed that CAV2-Cre spread was restricted to the LS (Supplementary Figure S3). Consistent with previous findings, LS-projecting vHPC cells were not uniformly distributed within the vHPC. mCherry-positive cell bodies were abundant in the ventral CA 3 and CA 1 region, but absent in the dentate gyrus (Figure 2b and Supplementary Figure S4) (Canteras and Swanson, 1992; Risold and Swanson, 1996,1997). Whole-cell patch-clamp recordings on hippocampal slices confirmed that CNO activated the hM3D receptor, depolarizing the membrane potential and increasing the firing rate of hM3D-expressing hippocampal pyramidal cells (Supplementary Figure S1).
Mice infused with CAV2-Cre into the LS and AAV8-hSynFLEX-hM3D-mCherry or AAV8-hSyn-FLEX-GFP into the vHPC were treated with $\mathrm{CNO}$ and underwent behavioral tests of anxiety. In the EPM, CNO-treated hM3D mice showed increased \% open arm time (Unpaired $t$-test, $t_{(19)}=4.77 p=0.0001$, Figure $2 \mathrm{c}$ and $\mathrm{f}$ for position plots) and open arm entries (Unpaired $t$-test, $t_{(19)}=2.56 p=0.01$, Figure 2d) compared to CNO-treated control mice. This phenotype was not due to increased locomotion, as hM3D and control mice did not display differences in locomotion in the EPM (Figure 2e). In the OF test, no significant differences between the groups were observed in \% time spent in the center and center entries (Figure $2 \mathrm{~g}$ and $\mathrm{h}$ and $\mathrm{k}$ for position plots) and locomotion (Figure $2 \mathrm{i}$ and $\mathrm{j}$ ). The absence of effect in the OF test prompted us to further examine the mice in additional tests of anxiety (SA and NSF tests). In the SA test, CNO-treated hM3D mice displayed increased \% open alley time in comparison with vehicletreated hM3D mice or CNO-treated control mice (RM twoway ANOVA, $\mathrm{F}_{(1,}{ }_{18)}=5.763 p=0.02$, Supplementary Figure S5A). No alterations were observed in the total open alley entries ( $\mathrm{RM}$ two-way ANOVA, $\mathrm{F}_{(1,18)}=4.3 p=0.05$, Supplementary Figure S5B). In the NSF test, CNO-treated hM3D mice showed reduced latency to feed in a novel environment ( $\mathrm{RM}$ two-way ANOVA, $\mathrm{F}_{(1,18)}=5.483 p=0.03$, Figure 2l). The reduced latency to feed was not due to changes in hunger, since hM3D and control mice showed no difference in home cage food consumption (Figure $2 \mathrm{~m}$ ). Thus, three different tests of anxiety confirmed that activation of LS-projecting vHPC cells is sufficient to cause a decrease in anxiety. Next, we explored whether the LSprojecting vHPC cells could modulate anxiety in a bidirectional manner.

\section{hM4D-Mediated Inhibition of LS-Projecting vHPC Cells Increases Anxiety}

On the basis of ability of LS-projecting vHPC cells to suppress anxiety when stimulated, we tested whether inhibition of LS-projecting vHPC cells could increase anxiety. Again, the combined use of CAV2-Cre and AAV8FLEX-hM4D-mcherry allowed us to inhibit LS-projecting vHPC cells and determine their specific contributions to anxiety (Figure 2a). In the EPM, CNO-treated hM4D mice displayed significantly lower \% open arm time (Unpaired $t$-test, $\mathrm{t}_{(24)}=3.96 p=0.0006$, Figure $3 \mathrm{a}$ and $\mathrm{d}$ for position plots) and open arm entries (Unpaired $t$-test, $\mathrm{t}_{(24)}=2.568$ $p=0.01$, Figure $3 \mathrm{~b}$ ) compared to the control mice, reflecting an increase in anxiety. Notably, CNO treatment in the hM4D 

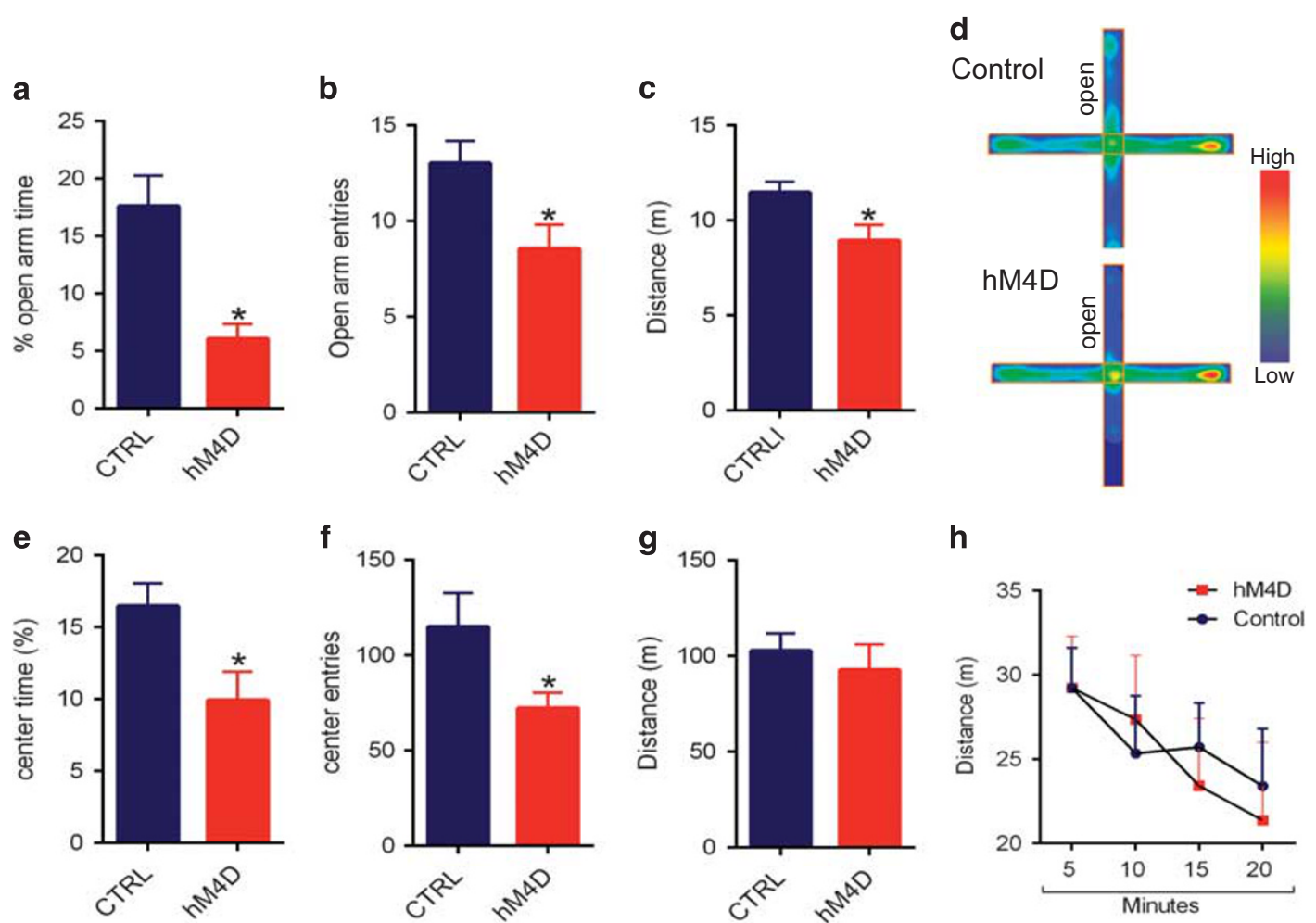

h
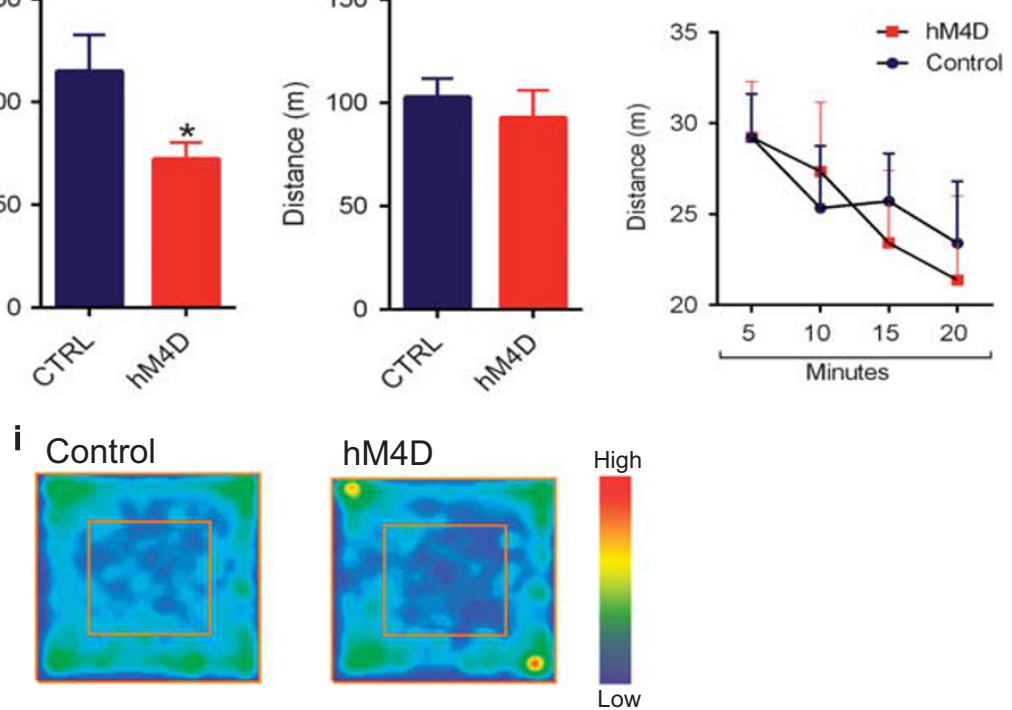

Figure 3 hM4D-mediated inhibition of LS-projecting VHPC cells increases anxiety. (a) hM4D-mice displayed a decreased \% open arm time in the EPM after CNO injection $(* p=0.0006$ ). (b) hM4D mice displayed decreased open arm entries in the EPM after CNO injection (*p $=0.00 \mathrm{l}$ ). (c) hM4D mice displayed a decreased level of locomotor activity in the EPM after CNO injection $(* p=0.0 \mathrm{I})$. (d) Heat-map plots show the averaged cumulative time spent in different parts of the EPM. red = more time, blue $=$ less time. (e and f) hM4D mice showed a decreased \% time spent in the center $(* p=0.03)$ and center entries in the OF after CNO injection (*p=0.03). (g) CTRL and hM3D mice showed similar levels of locomotor activity in the OF after CNO injection. (h) Examination of locomotor activity in 5 min intervals revealed no significant differences between hM4D and CTRL mice. (i) Heat-map plots show the averaged cumulative time spent in different parts of the OF. red = more time, blue $=$ less time. $n=13$ for CTRL and 13 for hM3D mice.

mice resulted in a decrease in overall exploratory locomotion during the EPM test (Unpaired $t$-test, $\mathrm{t}_{(24)}=2.53 p=0.01$, Figure 3c). While the decreased locomotion can act as a confounding factor when assessing anxiety, it may be attributed to anxiogenic effects restricting exploration to the closed arms. To further examine the effects of inhibiting the LS-projecting VHPC cells on anxiety and locomotion, we tested mice in the OF test. Consistent with the EPM results, the OF test revealed a similar anxiety-promoting effect of CNO treatment in the hM4D mice. hM4D mice showed a decrease in both \% center time (Unpaired $t$-test, $t_{(18)}=2.32$ $p=0.03$, Figure $3 \mathrm{e}$ ) and center entries (Unpaired $t$-test, $t_{(18)}=2.53 p=0.03$, Figure $3 \mathrm{f}$ and $\mathrm{i}$ for position plots) relative to the control. Importantly, no change in total locomotor activity was observed in the OF task (Figure $3 \mathrm{~g}$ and $\mathrm{h}$ ). Last, mice were tested in the SA using a within-subject design where both control and hM4D mice were injected with vehicle or CNO. No significant differences among the groups were observed in \% open alley time or open alley entries (Supplementary Figure S5D and S5E). In summary, these data demonstrated that the LS-projecting vHPC cells have the capacity to modulate anxiety-related behaviors in a bidirectional manner suggestive of an importance of this pathway in maintaining appropriate levels of anxiety.

\section{Activation and Inhibition of mPFC-Projecting vHPC Cells Cause an Increase and Decrease in Anxiety, Respectively}

Recent studies found that anxiety-related firing is selectively increased in vHPC neurons projecting to the MPFC (Ciocchi et al, 2015) and that optogenetic inhibition of vHPC inputs to the mPFC reduced anxiety (Padilla-Coreano et al, 2016). However, it has not been determined whether stimulating vHPC inputs to the $\mathrm{mPFC}$ is sufficient to increase anxiety. Furthermore, LS-projecting cells and mPFC-projecting cells 
a

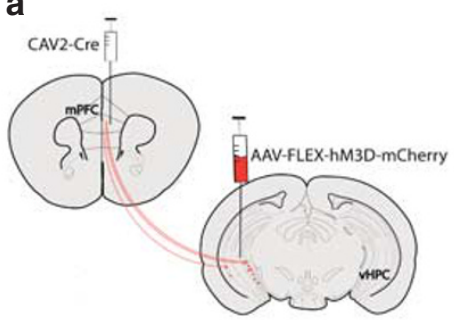

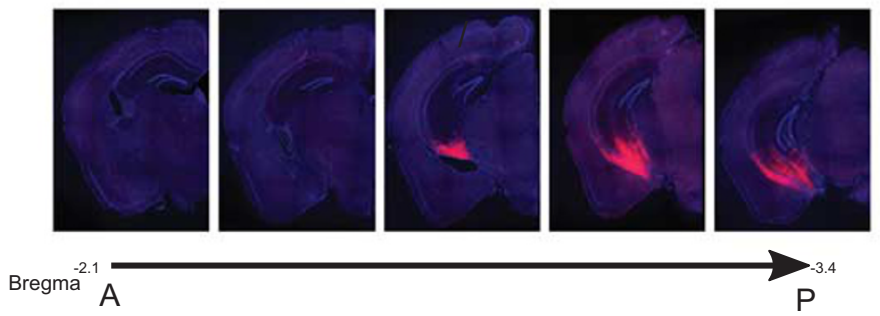

e

f g

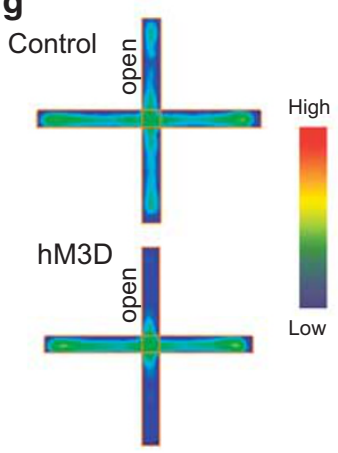

h

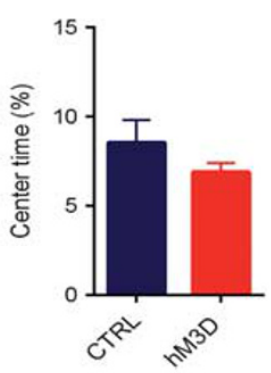

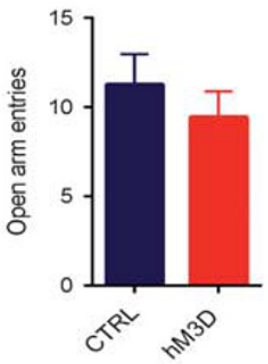

i

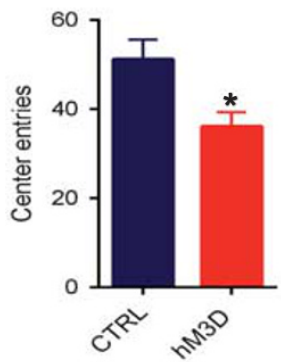

j

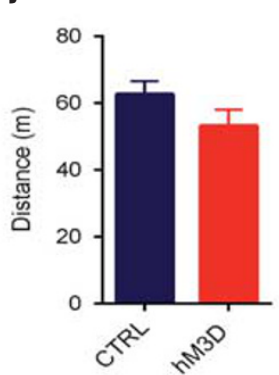

k
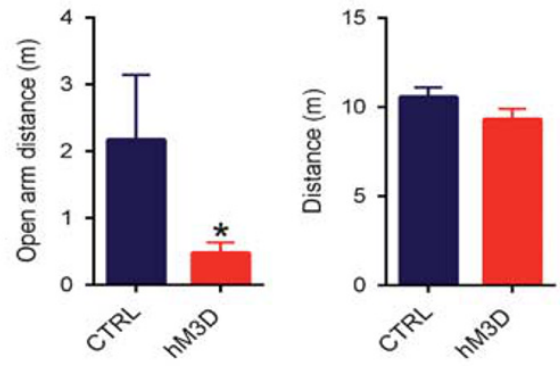

High

I Control
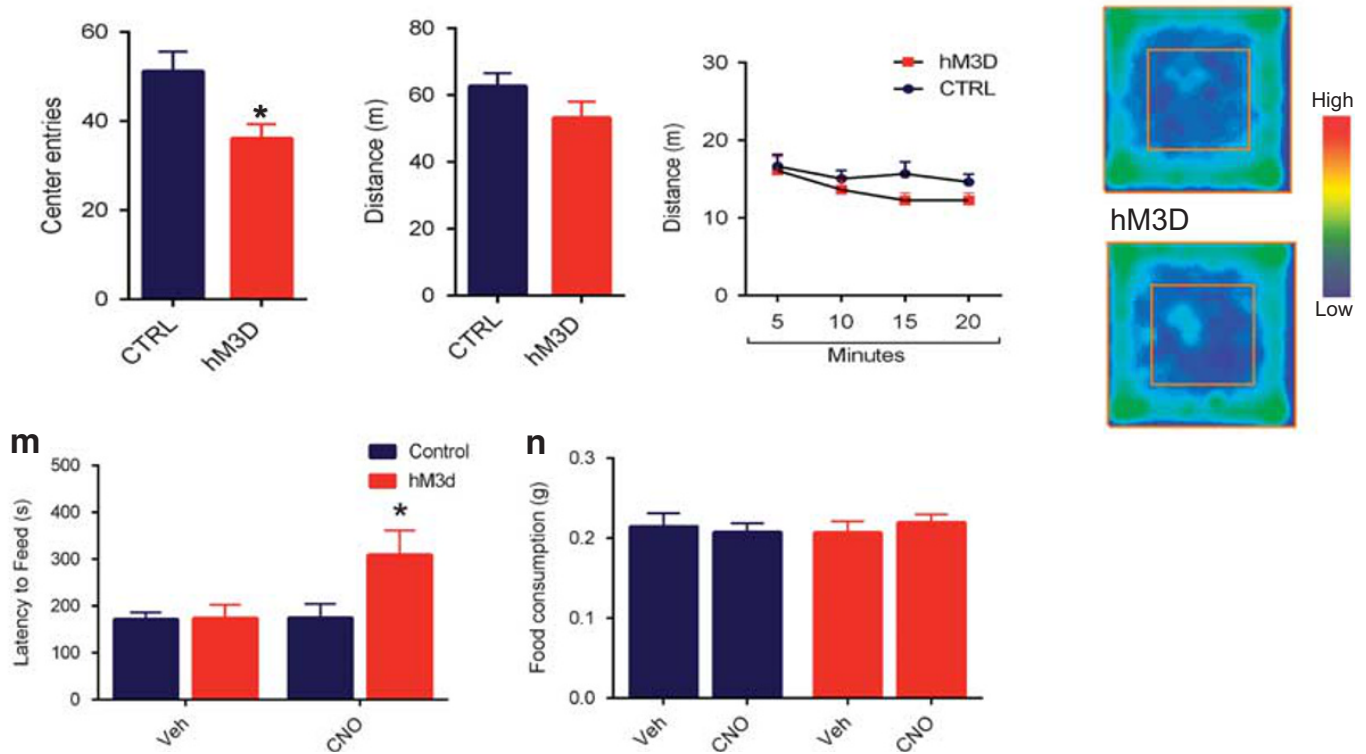

Figure 4 hM3D-mediated activation of mPFC-projecting vHPC cells increases anxiety. (a) mPFC-projecting vHPC cells were targeted by injecting the retrogradely propagating CAV2-Cre virus into the mPFC and injecting AAV8FLEXhM3DmCherry into the vHPC. (b) Representative images from AAV8FLEXhM3DmCherry mice showed hM3D-mCherry expression in MPFC-projecting VHPC cells along the anterior-posterior axis. (c-e) hM3D mice displayed a decreased \% open arm time $(* p=0.002)$ and open arm distance $(* p<0.05)$ without change in the number of open arm entries. $(f) C T R L$ and hM3D mice showed similar levels of locomotor activity measured as a total distance traveled in the EPM. (g) Heat-map plots show the averaged cumulative time spent in different parts of the EPM. red $=$ more time, blue $=$ less time. ( $h$ and i) hM3D mice displayed a decreased center entries ( $* p=0.0 \mathrm{I}$ ) after $\mathrm{CNO}$ injection, without changes in \% center time. (j) CTRL and hM3D mice showed similar levels of locomotor activity measured as a total distance traveled in the OF. (k) Examination of locomotor activity in 5 min intervals revealed no significant differences between hM3D and CTRL mice. (I) Heat-map plots show the averaged cumulative time spent in different parts of the OF. red = more time, blue =less time. $(\mathrm{m}) \mathrm{CNO}$ increased the latency to feed in hM3D mice but not in CTRL mice in the NSF test (RM two-way ANOVA shows a significant effect of the treatment $F_{(1,16)}=5.37$ I * $p=0.034$, followed by Bonferroni post-test $p=0.004$ ). (n) No alteration the food consumption was observed among the groups after the NFST. $n=7$ for CTRL and II for hM3D mice.

may represent two functionally distinct cell groups within the vHPC, regulating anxiety in opposite directions. To address this, we first examined the effect of activating $\mathrm{mPFC}$ projecting vHPC cells on anxiety using CAV2-Cre-mediated expression of hM3D (Figure 4a).
In contrast to the LS-projecting cells, mPFC-projecting vHPC cells were found almost exclusively in the CA1 (Figure $4 \mathrm{~b}$ and Supplementary Figure S4), indicative of an anatomical segregation between LS- and mPFC-projecting vHPC cells. Mice infused with CAV2-Cre (into the mPFC) 

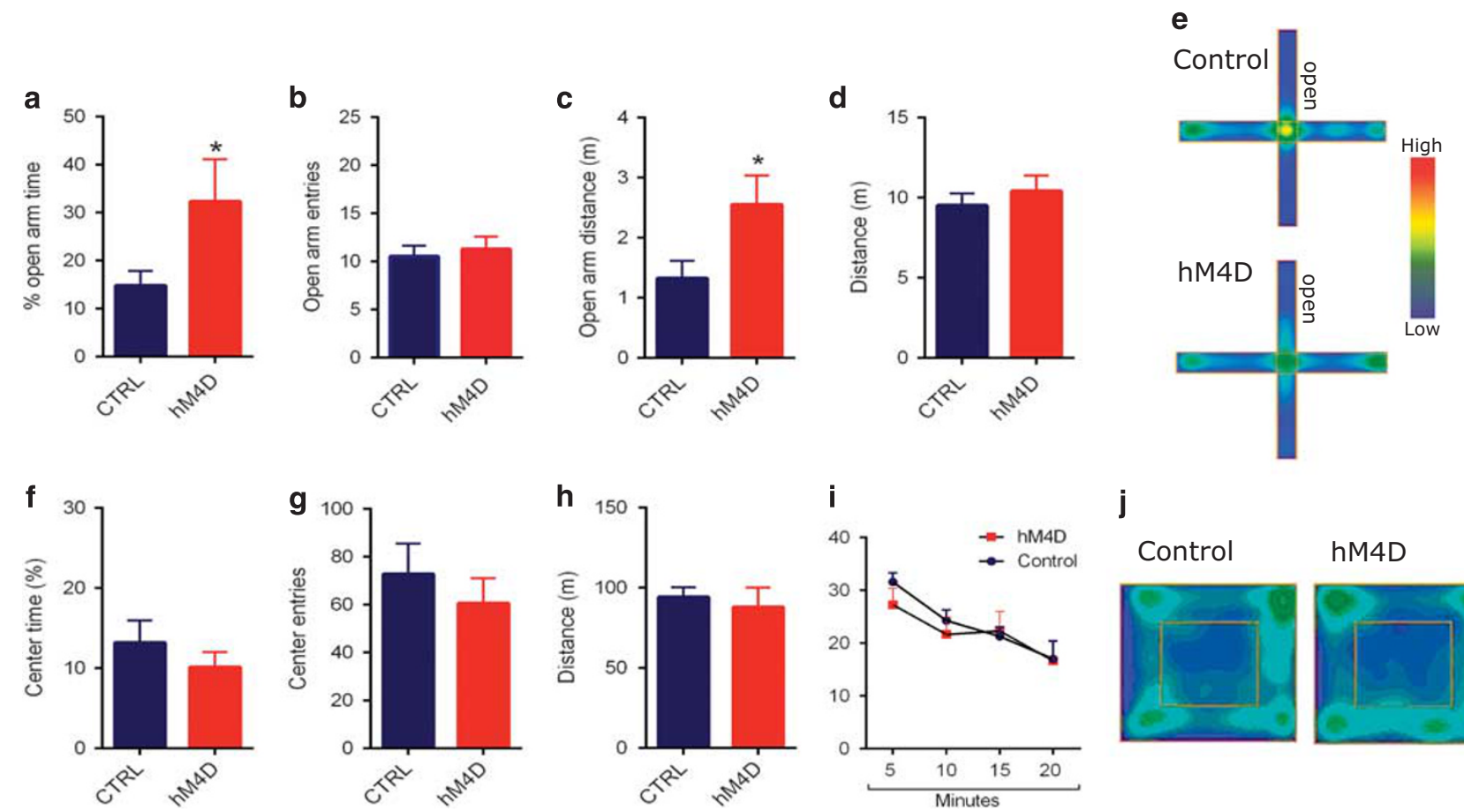

j
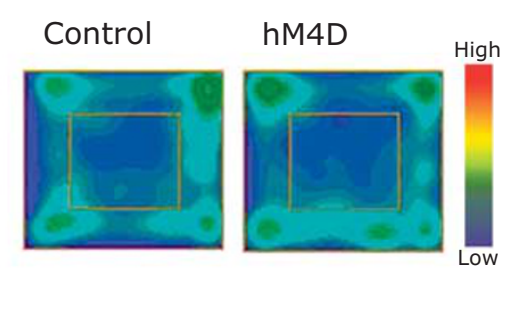

Figure 5 hM4D-mediated inhibition of mPFC-projecting VHPC cells decreases anxiety. (a-c) hM4D mice displayed an increased \% open arm time $(* p=0.02)$ and open arm distance $(* p=0.03)$ without change in the number of open arm entries. (d) CTRL and hM4D mice showed similar levels of locomotor activity measured as a total distance traveled in the EPM. (e) Heat-map plots show the averaged cumulative time spent in different parts of the EPM. red $=$ more time, blue $=$ less time. $(f-h) C T R L$ and $h M 4 D$ mice showed similar levels of $\%$ center time, center entries, and locomotor activity in the OF test. (i) Examination of locomotor activity in 5 min intervals revealed no significant differences between hM4D and CTRL mice. (j) Heat-map plots show the averaged cumulative time spent in different parts of the OF. red = more time, blue $=$ less time.

and AAV8-hSyn-FLEX-hM3D-mCherry or AAV8-hSynFLEX-GFP (into the vHPC) underwent the same behavioral assays after an IP injection of CNO. In the EPM test, CNO-treated hM3D mice showed decreased \% open arm time (Unpaired $t$-test, $t_{(16)}=2.32 p=0.02$, Figure $4 \mathrm{c}$ and $\mathrm{g}$ for position plots) and distance traveled in open arm (Unpaired $t$-test, $t_{(16)}=2.0 p<0.05$, Figure 4e) without change in the number of open arm entries (Figure 4d), reflecting an increase in anxiety. Importantly, no alteration in the total locomotor activity was observed throughout the EPM test (Figure 4f). In the OF test, CNO-treated hM3D mice showed a decrease in both center entries (Unpaired $t$ test, $t_{(14)}=2.32$ $p=0.01$, Figure $4 \mathrm{i}$ and 1 for position plots) and distance traveled in the center (Unpaired $t$ test, $t_{(14)}=2.78 p=0.01$, data not shown) and no change in \% time spent in the center and total locomotor activity (Figure $4 \mathrm{~h}, \mathrm{j}$ and $\mathrm{k}$ ), indicating an increase in anxiety-like behaviors. In the NSF test, CNOtreated hM3D mice displayed an increased latency to feed (RM two-way ANOVA, $\mathrm{F}_{(1,16)}=5.371 P=0.03$, Figure $4 \mathrm{~m}$ ). The increased latency to feed was not due to changes in hunger since $\mathrm{CNO}$ and vehicle group showed no difference in home cage food consumption (Figure $4 \mathrm{n}$ ). Consistent with the previous study by Padilla-Coreano et al, we found evidence for the anxiety-reducing effecting of inhibiting mPFC-projecting vHPC cells. In the EPM test, CNO-treated hM4D mice showed increased \% open arm time (Unpaired $t$ test, $t_{(16)}=2.04 p=0.02$, Figure $5 \mathrm{a}$ and e for position plots) and distance traveled in open arm (Unpaired $t$-test, $t_{(16)}=2.27 p=0.03$, Figure $5 c$ ). In the OF test, no significant differences between the groups were observed in \% time spent in the center and center entries (Figure $5 \mathrm{f}-\mathrm{h}$ ) and locomotion (Figure 5i). Altogether, these findings indicate that the vHPC houses two functionally distinct cell populations regulating anxiety in opposite directions: the LS-projecting cells, which suppress anxiety, and the mPFC-projecting cells, which promote anxiety.

\section{LS- and mPFC-Projecting vHPC Cells Represent Anatomically Discrete Neuronal Populations that Reside in Relatively Distinct Areas of the vHPC}

While LS- and mPFC-projecting vHPC cells were targeted based on their specific postsynaptic connections, these vHPC cells may collateralize and project to other brain areas simultaneously. To examine the distribution of collateral axon terminals of LS- and mPFC-projecting vHPC cells, we immunostained the tissue sections with the anti-mCherry antibody. As expected, mCherry-positive axonal terminals of the LS-projecting vHPC cells were densest in the LS (Figure $6 \mathrm{a}$ and $\mathrm{b}$ ). While much less abundant, mCherry-positive terminals were also found in the dorsal CA1, basolateral amygdala (BLA), and mPFC, suggesting that a small proportion of the LS-projecting vHPC cells innervate not only the LS but also other downstream targets via collateralized axons (Figure 6b). Similarly, mCherrypositive axonal terminals of mPFC-projecting vHPC cells were densest in the $\mathrm{mPFC}$, with much less dense terminal fields found in other known downstream targets of the vHPC, including the LS (Figure 6b). 
a

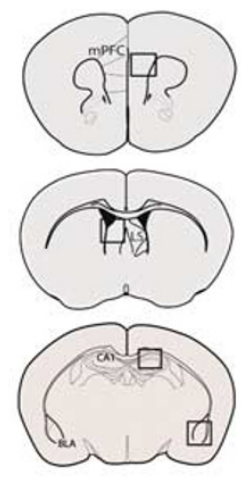

b

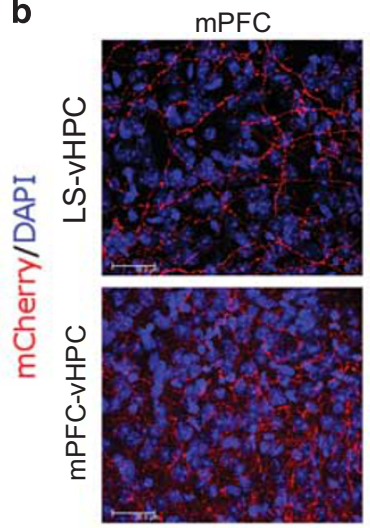

dLS

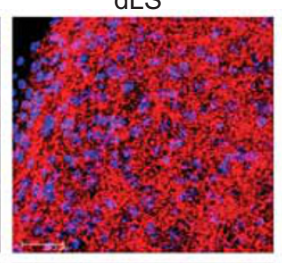

C

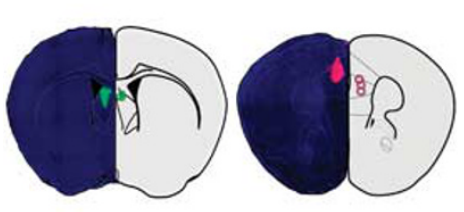

e

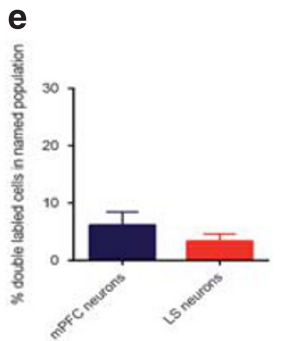

Injection sites

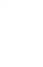

d

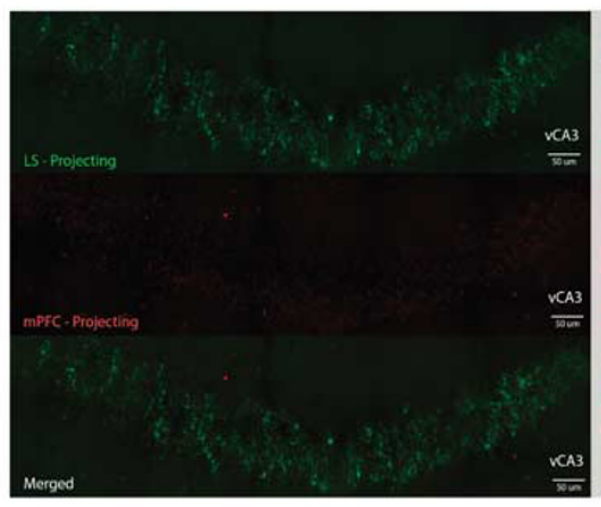

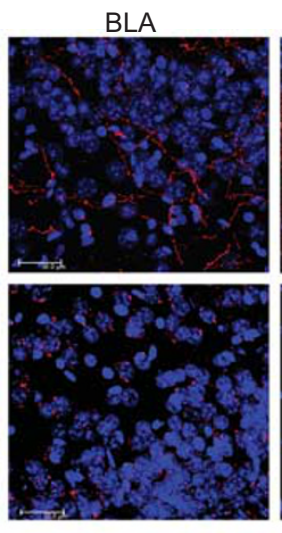

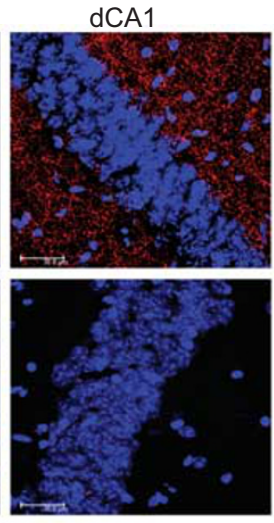

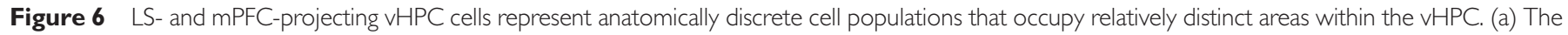

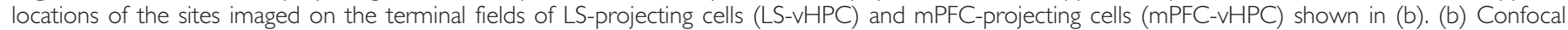

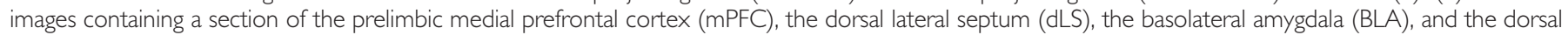

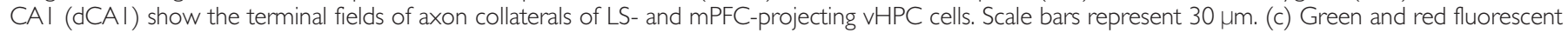

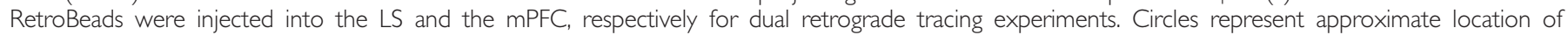

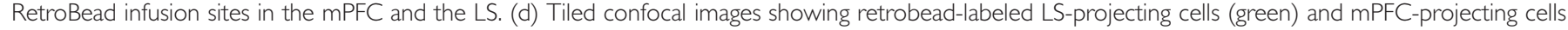

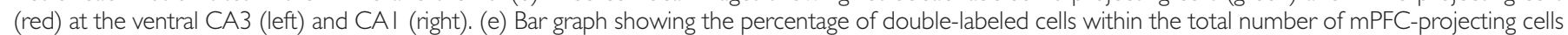
(blue) and LS-projecting cells (red) in the ventral CAI ( $n=3$ mice).

The largely distinct postsynaptic connectivity of LS- and mPFC-projecting cells lent further support that they represent anatomically separate cell populations. We therefore investigated how the LS- and MPFC projecting cells are distributed within the vHPC and to what extent the two cell populations overlap with each other. For dual retrograde tracing experiments, green and red fluorescent retrogradelytransported microspheres (Retrobeads) were injected ipsilaterally into the LS and MPFC to label LS- and PFC-projecting vHPC cells, respectively (Figure 6c). Consistent with CAV2Cre-mediated retrograde labeling of LS-projecting vHPC cells, retrobead-labeled LS-projecting cells were found most abundant in the ventral CA3 pyramidal layer, less in the ventral CA1 pyramidal cell layers, and absent in the dentate gyrus (Figure 6d). In contrast, retrobead-labeled mPFCprojecting cells were found in the ventral CA1 but absent in the CA3 and the dentate gyrus (Figure 6d). Within the vHPC, 3.3\% of the retrobead-labeled LS-projecting cells were found to be mPFC-projecting, and $6.1 \%$ of the retrobeadlabeled mPFC-projecting vHPC cells were LS-projecting (Figure 6e). Thus, mPFC- and LS-projecting cells represent anatomically discrete neuronal populations that occupy relatively distinct areas within the vHPC, playing opposite roles in regulating anxiety.

\section{DISCUSSION}

Neural inputs arising from the vHPC to the LS and mPFC have been implicated in anxiety. However, the specific role of these vHPC-originated signals in anxiety has not been directly compared, as previous lesion and pharmacological manipulation approach have been limited in discerning celltype or pathway-specific contribution. Combining a chemogenetic neuron manipulation approach with Cre-expressing retrograde viral vectors has allowed us to overcome longstanding technical limitations to target specific neural elements of the septohippocampal pathway and determine their behavioral contribution in vivo. First, we found that acute inactivation of the entire vHPC excitatory cells produced robust anxiolytic effects, consistent with previous findings that temporary or permanent inactivation of the vHPC, but not the dorsal hippocampus, reduce anxiety- 
related behaviors. Next, we demonstrated that a subset of vHPC cells projecting to the LS modulate anxiety in a bidirectional manner, where activation of LS-projecting vHPC cells decreases anxiety and inhibition of the same cell population produces opposite anxiety-promoting effect. The anxiety-reducing role of the LS-projecting vHPC cells was in sharp contrast to that of mPFC-projecting vHPC cells, the activity of which increased anxiety. These findings support the idea that hippocampal control of anxiety is mediated by a balance between LS- and mPFC-projecting cell activities.

\section{Anxiolytic Function of LS-Projecting vHPC Cells}

Compared to its anxiety-promoting role, the anxiolytic function of the vHPC and its mechanism of action are much less understood. Previous studies have shown that vHPC lesion increases corticotropin-releasing hormone messenger RNA (mRNA) expression in the paraventricular nucleus (PVN) and extends the duration of corticosterone response to physical restraint or exposure to anxietyprovoking stimuli, suggesting that the vHPC suppresses stress responses by down-regulating the HPA axis (Herman et al, 1995, 2003; Herman and Cullinan, 1997; Mueller et al, 2004; Radley and Sawchenko, 2011). There is, however, no direct projection from the vHPC cells to the PVN (Cullinan et al, 1993), which suggests that a subset of vHPC cells expressing corticosterone receptors would exert a polysynaptic inhibition on PVN neuron activity, thereby downregulating the HPA axis.

A plausible idea is that the LS may mediate this polysynaptic inhibition as an intermediary structure connecting the vHPC to the PVN (Mueller et al, 2006). In keeping, it has recently been shown that a subset of GABA neurons in the LS characterized by corticotropin releasing factor receptor 2 expression innervate the anterior hypothalamic nucleus (AHN), a GABAergic structure that inhibits the PVN (Anthony et al, 2014). Using an in vivo optogenetic approach, the study found that stimulating the AHNprojecting LS GABA neurons increased corticosterone level and produced persistent anxiety-related behaviors. This suggests that the AHN-projecting LS GABA neurons promote neuroendocrine and behavioral aspects of anxiety by disinhibiting the HPA axis. While the contribution of vHPC in controlling the LS-AHN pathway remains to be determined, the vHPC may preferentially innervate a distinct population of LS GABA neurons that in turn inhibit the AHN-projecting LS GABA neurons. Thus, the activation of LS-projecting vHPC cells may down-regulate the HPA axis by disinhibiting the AHN.

In addition to potential neuroendocrine inputs to the LSprojecting vHPC cells, glutamatergic inputs from the ventral dentate gyrus (DG) may also contribute to the anxiolytic role of LS-projecting vHPC cells. LS-projecting vHPC cells were found most abundant in the ventral CA3 subregion and less in the ventral CA1 pyramidal cell layers. However, it is currently unclear how these LS-projecting ventral CA1 and CA3 cells differently control anxiety. The ventral CA3 pyramidal cells receive glutamatergic mossy fiber inputs from the ventral, but not dorsal, DG through the trisynaptic pathway in a region-specific manner. Notably, a recent study by Kheirbek et al found that the activation of the ventral DG resulted in a decrease in anxiety-related behaviors without affecting contextual learning (Kheirbek et al, 2013). Because the granule cells in the ventral DG do not directly project to extrahippocampal structures, but instead drive hippocampal outputs by innervating the ventral CA3 subregion, the anxiolytic effect of the ventral DG activation may be mediated by the LS-projecting CA3 cells. Future work will have to determine this possibility with pathway-specific approaches targeting DG inputs to the CA3 within the vHPC.

\section{Anxiogenic Function of the mPFC-Projecting vHPC Cells}

Converging data suggest that the vHPC conveys an anxiogenic signal to the $\mathrm{mPFC}$ via synchronized theta rhythms. Theta power in both vHPC and mPFC increases when animals are exposed to anxiety-provoking environments such as EPM and OF (Adhikari et al, 2010). The observed theta power correlation between the two structures increased right before animals escaped to safer closed arms of the EPM and decreased right before animals entered open arm space. Consistently, recent studies using in vivo unit recording and optogenetic inhibition found that anxietyrelated firing is selectively increased in vHPC neurons projecting to the mPFC (Ciocchi et al, 2015) and that optogenetic inhibition of vHPC inputs to the $\mathrm{MPFC}$ reduced anxiety-related behaviors (Padilla-Coreano et al, 2016). These findings, together with ours, suggest that vHPC inputs to the mPFC convey anxiogenic signals and may promote avoidance behaviors over approach behaviors in a novel environment. In addition, it should be noted that the anxiogenic effects of mPFC-projecting vHPC cells may be driven by amygdala inputs to the vHPC. Recently, Felix Ortiz et al found that activating BLA inputs to the CA1 subregion acutely and reversibly increases anxiety-related behaviors (Felix-Ortiz et al, 2013). This indicates that the ventral CA1 cells innervated by the BLA inputs represent an anxiogenic population and may potentially overlap with the mPFC-projecting vHPC cells. While it remains to be determined by retrograde tracing experiments, it is plausible that during threatening situations, the BLA may excite preferentially the mPFC-projecting vHPC cells over the LSprojecting vHPC cells to promote anxiety.

\section{Anatomical Segregation between LS- and mPFC- Projecting vHPC Cells}

The use of CAV2-Cre in combination with the Creresponsive AAV vectors allowed us to activate (by hM3D) or inhibit (by hM4D) vHPC cells that project to the LS or the mPFC (van Gestel et al, 2014). It should be noted, however, that retrogradely-targeted vHPC cells send axon collaterals to multiple target structures. For example, the axon terminals of the LS-projecting vHPC cells were most abundant in the LS, but they were also found in the dCA1 and the BLA to lesser degree. Therefore, behavioral changes resulting from activating or inhibiting the LS-projecting vHPC cells likely reflect the combined effects of altering multiple axon collaterals.

Our retrograde tracing revealed that (anxiety-promoting) mPFC-projecting cells and (anxiety-suppressing) LSprojecting cells are largely discrete populations of neurons occupying relatively distinct areas of the vHPC; $\mathrm{mPFC}$ projecting cells are located almost exclusively in the ventral 
CA1, while LS-projecting cells in both the ventral CA3 and CA1 (Figure 6). In addition, we identified a small subset of vHPC cells $(3.3 \%$ of total LS- projecting cells and $6.1 \%$ of total mPFC-projecting cells) that send collateralized axon projections to the LS and the MPFC (Figure 6b). The function of these small number of vHPC cells projecting simultaneously to the LS and MPFC is currently unknown and could not be addressed in the present study as systemic administration of $\mathrm{CNO}$ activated both projections simultaneously. Therefore, more specific optogenetic experiments, where vHPC axon terminals are activated or inhibited through optic fibers implanted over the LS and the mPFC, will be needed to determine the relative contribution of each pathway. Caution, however, must be exercised when illuminating the vHPC terminals in the LS as hippocampal axon bundles projecting to other brain areas pass through the septum, and it is technically challenging to restrict light illumination only to vHPC terminals in the LS without illuminating the axon bundles traveling through the septum (but see also (Sweeney and Yang, 2015)).

Anxiety-related firing of the ventral CA1 cells differs depending on their projection targets, even among the cells sharing the mPFC as a common postsynaptic target (Ciocchi et al, 2015). For example, the ventral CA1 cells projecting exclusively to the mPFC were enriched with neurons displaying anxiety-related firing. Importantly, however, this enrichment was not observed in other neighboring CA1 cells that simultaneously project to both mPFC and nucleus accumbens. A similar type of target-specific firing may also be found among the LS-projecting and mPFC-projecting CA1 cells. The ventral CA1 cells projecting to both the LS and the MPFC may have different anxiety-related firing from the ventral CA1 cells projecting exclusively to the LS or the mPFC. Thus, it would be of great interest to compare the task-related firing pattern of the ventral CA1 cells that project to either the LS or MPFC, or both.

While the behavioral effects of manipulating LS- and mPFC-projecting vHPC cells were mostly consistent across different anxiety tests, there were a few cases of inconsistencies. For example, the effects of activating LS- and mPFC-vHPC cells were found in the EPM, but not in the OF test. Although both EPM and OF are widely used as tasks that measure anxiety-related behaviors, the EPM is regarded as the most sensitive test of anxiety and currently the firstchoice for screening anxiolytic drugs (Ramos, 2008; Fraser et al, 2010). Moreover, EPM and OF tests may not assess the same psychobiological phenomenon. Thus, the differences between the OF and EPM results in our study may reflect subtle differences in anxiety-related behaviors associated with different vHPC pathways.

Anxiety response has a protective role in animals facing potential threats. However, inappropriate and exaggerated anxiety responses lead to maladaptive conditions shared by various psychiatric disorders. Our results suggest that the vHPC contributes to such processes by monitoring the novelty and aversiveness of the environment and broadcasting contextual information to the LS and mPFC. Adjusting the balance between the activities of these two vHPC neuron populations may represent an important mechanism for regulating the level of anxiety and inform novel therapeutic strategies tailored to hippocampal dysfunction observed in anxiety disorders.

\section{FUNDING AND DISCLOSURE}

The authors declare no conflict of interest.

\section{ACKNOWLEDGMENTS}

JCK was supported by the Natural Sciences and Engineering Council of Canada (NSERC) Discovery grant (MOP 491009) and a CIHR grant (MOP 496401). BAR was supported by NSERC Discovery grant (MOP 04947). GMP was funded by the postdoctoral fellowship from the Brazilian National Council for Scientific and Technological Development (CNPq). RN was funded by the Ontario Mental Health Foundation (OMHF) scholarship.

\section{REFERENCES}

Adhikari A, Topiwala MA, Gordon JA (2010). Synchronized activity between the ventral hippocampus and the medial prefrontal cortex during anxiety. Neuron 65: 257-269.

Adhikari A, Topiwala MA, Gordon JA (2011). Single units in the medial prefrontal cortex with anxiety-related firing patterns are preferentially influenced by ventral hippocampal activity. Neuron 71: 898-910.

Albert DJ, Walsh ML (1982). The inhibitory modulation of agonistic behavior in the rat brain: a review. Neurosci Biobehav Rev 6: 125-143.

Anthony TE, Dee N, Bernard A, Lerchner W, Heintz N, Anderson DJ (2014). Control of stress-induced persistent anxiety by an extra-amygdala septohypothalamic circuit. Cell 156: 522-536.

Armbruster BN, Li X, Pausch MH, Herlitze S, Roth BL (2007). Evolving the lock to fit the key to create a family of $G$ proteincoupled receptors potently activated by an inert ligand. Proc Natl Acad Sci 104: 5163-5168.

Bannerman DM, Grubb M, Deacon RMJ, Yee BK, Feldon J, Rawlins JNP (2003). Ventral hippocampal lesions affect anxiety but not spatial learning. Behav Brain Res 139: 197-213.

Brady JV, Nauta WJH (1955). Subcortical mechanisms in emotional behavior: the duration of affective changes following septal and habenular lesions in the albino rat. J Comp Physiol Psychol 48: 412-420.

Calhoon GG, Tye KM (2015). Resolving the neural circuits of anxiety. Nat Neurosci 18: 1394-1404.

Campeau S, Watson SJ (1997). Neuroendocrine and behavioral responses and brain pattern of c-fos induction associated with audiogenic stress. J Neuroendocrinol 9: 577-588.

Canteras NS, Swanson LW (1992). Projections of the ventral subiculum to the amygdala, septum, and hypothalamus: a PHAL anterograde tract-tracing study in the rat. J Comp Neurol 324: 180-194.

Ciocchi S, Passecker J, Malagon-Vina H, Mikus N, Klausberger T (2015). Selective information routing by ventral hippocampal CA1 projection neurons. Science 348: 560-563.

Cullinan WE, Herman JP, Watson SJ (1993). Ventral subicular interaction with the hypothalamic paraventricular nucleus: Evidence for a relay in the bed nucleus of the stria terminalis. J Comp Neurol 332: 1-20.

Deacon RMJ (2013). The successive alleys test of anxiety in mice and rats. J Vis Exp 76: 1-7.

Degroot A, Kashluba S, Treit D (2001). Septal GABAergic and hippocampal cholinergic systems modulate anxiety in the plus-maze and shock-probe tests. Pharmacol Biochem Behav 69: 391-399.

Duncan GE, Knapp DJ, Breese GR (1996). Neuroanatomical characterization of Fos induction in rat behavioral models of anxiety. Brain Res 713: 79-91.

Felix-Ortiz AC, Beyeler A, Seo C, Leppla CA, Wildes CP, Tye KM (2013). BLA to vHPC inputs modulate anxiety-related behaviors. Neuron 79: 658-664. 
Fraser LM, Brown RE, Hussin A, Fontana M, Whittaker A, O'Leary TP et al (2010). Measuring anxiety- and locomotionrelated behaviours in mice: a new way of using old tests. Psychopharmacology 211: 99-112.

Freund TF, Antal M (1988). GABA-containing neurons in the septum control inhibitory interneurons in the hippocampus. Nature 336: 170-173.

Grupe DW, Nitschke JB (2013). Uncertainty and anticipation in anxiety: an integrated neurobiological and psychological perspective. Nat Rev Neurosci 14: 488-501.

Hasler G, Fromm S, Alvarez RP, Luckenbaugh DA, Drevets WC, Grillon C (2007). Cerebral blood flow in immediate and sustained anxiety. J Neurosci 27: 6313-6319.

Herman JP, Cullinan WE (1997). Neurocircuitry of stress: central control of the hypothalamo-pituitary-adrenocortical axis. Trends Neurosci 20: 78-84.

Herman JP, Cullinan WE, Morano MI, Akil H, Watson SJ (1995). Contribution of the ventral subiculum to inhibitory regulation of the hypothalamo-pituitary-adrenocortical axis. J Neuroendocrinol 7: 475-482.

Herman JP, Figueiredo H, Mueller NK, Ulrich-Lai Y, Ostrander MM, Choi DC et al (2003). Central mechanisms of stress integration: Hierarchical circuitry controlling hypothalamo-pituitary-adrenocortical responsiveness. Front Neuroendocrinol 24: 151-180.

Kheirbek MA, Drew LJ, Burghardt NS, Costantini DO, Tannenholz L, Ahmari SE et al (2013). Differential control of learning and anxiety along the dorsoventral axis of the dentate gyrus. Neuron 77: 955-968.

Kiss J, Patel AJ, Baimbridge KG, Freund TF (1990). Topographical localization of neurons containing parvalbumin and choline acetyltransferase in the medial septum-diagonal band region of the rat. Neuroscience 36: 61-72.

Kjelstrup KG, Tuvnes FA, Steffenach H-A, Murison R, Moser EI, Moser M-B (2002). Reduced fear expression after lesions of the ventral hippocampus. Proc Natl Acad Sci USA 99: 10825-10830.

Kremer EJ, Boutin S, Chillon M, Danos O (2000). Canine adenovirus vectors: an alternative for adenovirus-mediated gene transfer. J Virol 74: 505-512.

McHugh SB, Deacon RMJ, Rawlins JNP, Bannerman DM (2004). Amygdala and ventral hippocampus contribute differentially to mechanisms of fear and anxiety. Behav Neurosci 118: 63-78.

McNaughton N (2006). The role of the subiculum within the behavioural inhibition system. Behav Brain Res 174: 232-250.

McNaughton N, Gray JA (2000). Anxiolytic action on the behavioural inhibition system implies multiple types of arousal contribute to anxiety. J Affect Disord 61: 161-176.

Menard J, Treit D (1996). Lateral and medial septal lesions reduce anxiety in the plus-maze and probe-burying tests. Physiol Behav 60: $845-853$.

Mongeau R, Miller GA, Chiang E, Anderson DJ (2003). Neural correlates of competing fear behaviors evoked by an innately aversive stimulus. J Neurosci 23: 3855-3868.

Mueller NK, Dolgas CM, Herman JP (2004). Stressor-selective role of the ventral subiculum in regulation of neuroendocrine stress responses. Endocrinology 145: 3763-3768.

Mueller NK, Dolgas CM, Herman JP (2006). Regulation of forebrain GABAergic stress circuits following lesion of the ventral subiculum. Brain Res 1116: 132-142.
Nguyen R, Morrissey MD, Mahadevan V, Cajanding J, Woodin MA, Yeomans JS et al (2014). Parvalbumin and GAD65 interneuron inhibition in the ventral hippocampus induces distinct behavioral deficits relevant to schizophrenia. J Neurosci 34: 14948-14960.

Niewiadomska G, Baksalerska-Pazera M, Riedel G (2009). The septo-hippocampal system, learning and recovery of function. Prog Neuro-Psychopharmacol Biol Psychiatry 33: 791-805.

Oler JA, Fox AS, Shelton SE, Rogers J, Dyer TD, Davidson RJ et al (2010). Amygdalar and hippocampal substrates of anxious temperament differ in their heritability. Nature 466: 864-868.

Padilla-Coreano N, Bolkan SS, Pierce GM, Blackman DR, Hardin WD, Garcia-Garcia AL et al (2016). Direct ventral hippocampalprefrontal input is required for anxiety-related neural activity and behavior. Neuron 89: 857-866.

Pesold C, Treit D (1996). Erratum: the neuroanatomical specificity of the anxiolytic effects of intra-septal infusions of midazolam (Brain Res. 710 (1996) 161-168) (BRES 11985). Brain Res 730: 243.

Pezzone MA, Lee W-S, Hoffman GE, Rabin BS (1992). Induction of c-Fos immunoreactivity in the rat forebrain by conditioned and unconditioned aversive stimuli. Brain Res 597: 41-50.

Radley JJ, Sawchenko PE (2011). A common substrate for prefrontal and hippocampal inhibition of the neuroendocrine stress response. J Neurosci 31: 9683-9695.

Radulovic J, Rühmann A, Liepold T, Spiess J (1999). Modulation of learning and anxiety by corticotropin-releasing factor (CRF) and stress: differential roles of CRF receptors 1 and 2. J Neurosci 19: 5016-5025.

Ramos A (2008). Animal models of anxiety: do I need multiple tests? Trends Pharmacol Sci 29: 493-498.

Risold PY, Swanson LW (1996). Structural evidence for functional domains in the rat hippocampus. Science 272: 1484-1486.

Risold PY, Swanson LW (1997). Connections of the rat lateral septal complex. Brain Res Brain Res Rev 24: 115-195.

Senba E, Matsunaga K, Tohyama M, Noguchi K (1993). Stressinduced c-fos expression in the rat brain: activation mechanism of sympathetic pathway. Brain Res Bull 31: 329-344.

Silveira MCL, Sandner G, Graeff FG (1993). Induction of Fos immunoreactivity in the brain by exposure to the elevated plus-maze. Behav Brain Res 56: 115-118.

Sweeney P, Yang Y (2015). Septum circuit that suppresses feeding. Nat Commun 6: 1-11.

Sylvers P, Lilienfeld SO, LaPrairie JL (2011). Differences between trait fear and trait anxiety: implications for psychopathology. Clin Psychol Rev 31: 122-137.

Trent NL, Menard JL (2010). The ventral hippocampus and the lateral septum work in tandem to regulate rats' open-arm exploration in the elevated plus-maze. Physiol Behav 101: 141-152.

van Gestel MA, Boender AJ, de Vrind VAJ, Garner KM, Luijendijk MCM, Adan RAH (2014). Recombinant adeno-associated virus: efficient transduction of the rat $\mathrm{VMH}$ and clearance from blood. PLoS One 9: e97639.

Wainer BH, Levey AI, Rye DB, Mesulam MM, Mufson EJ (1985). Cholinergic and non-cholinergic septohippocampal pathways. Neurosci Lett 54: 45-52.

Yadin E, Thomas E, Grishkat HL, Strickland CE (1993). The role of the lateral septum in anxiolysis. Physiol Behav 53: 1077-1083.

Supplementary Information accompanies the paper on the Neuropsychopharmacology website (http://www.nature.com/npp) 